\title{
Statistical Homogenization of Polycrystal Composite Materials with Thin Interfaces using Virtual Element Method
}

\author{
Marco Pingaro ${ }^{\mathrm{a}, *}$, Maria Laura De Bellis ${ }^{\mathrm{b}}$, Patrizia Trovalusci ${ }^{\mathrm{a}}$, Renato \\ Masiani ${ }^{\mathrm{a}}$ \\ ${ }^{a}$ Department of Structural and Geotechnical Engineering, Sapienza University of Rome, Via \\ Antonio Gramsci 53, 00197 Rome, Italy \\ ${ }^{b}$ Department of Engineering and Geology, Gabriele D'Annunzio University, Viale Pindaro \\ 42, 65122, Pescara, Italy
}

\begin{abstract}
Polycristalline materials with inter-granular phases are modern composite materials extremely relevant for a wide range of applications, including aerospace, defence and automotive engineering. Their complex microstructure is often characterized by stochastically disordered distributions, having a direct impact on the overall mechanical behaviour. In this context, within the framework of homogenization theories, we adopt a Fast Statistical Homogenization Procedure (FSHP), already developed in Pingaro et al. (2019), to reliably grasp the constitutive relations of equivalent homogeneous continua accounting for the presence of random internal structures. The approach, combined with the Virtual Element Method (VEM) used as a valuable tool to keep computational costs down, is here successfully extended to account for the peculiar microstructure of composites with polycrystals interconnected by thin interfaces. Numerical examples of cermet-like linear elastic composites complement the paper.

Keywords: Statistical Homogenization, Representative Volume Element, Random Composites, Grain Boundaries, Virtual Element Method

\footnotetext{
* Corresponding author

Email addresses: marco.pingaro@uniroma1.it (Marco Pingaro), marialaura.debellis@unich.it (Maria Laura De Bellis), patrizia.trovalusci@uniroma1.it (Patrizia Trovalusci), renato.masiani@uniroma1.it (Renato Masiani)
}

Preprint submitted to Elsevier

February 5, 2021 


\section{Introduction}

Modern composites materials are conceived to fulfil specific design requirements calling for the need of materials with increasingly high performances in terms of stiffness, strength, fracture toughness and lightness, among others. Special

5 attention has been devoted in the last decades to advanced ceramics, namely composites with ceramic (CMC) or metal matrix (MMC) [1, 2] which are gradually more used for a wide range of challenging applications, ranging from bioengineering, with the production of biocompatible ceramics for prosthesis and artificial organs [3], aerospace, defence, automotive [4] up to mechanical engineering for the production of wearing parts, seals, low weight components and fuel cells, and cutting tools with distinguished thermo-mechanical and wear properties [5]. Often ceramic composites exhibit a peculiar microstructure, characterized by polycrystals interconnected by a second-phase interphase of small thickness in comparison to the grain diameter. Consistently with [6], different combinations of materials lead to four kinds polycrystalline materials with intergranular phases: i) ceramic grains with brittle interphases; ii) ceramic grains and a metallic interphase; iii) metallic grains surrounded by ceramic interphases; iv) metallic grains surrounded by a layer of soft ductile material. In all cases, such materials show complex phenonema occurring at different scales of interest, ranging from the microscopic up to the macroscopic one, so that the elastic properties of the homogenized composite material is strongly influenced by the random distribution of grains, by the role played by the intergranular layer, as well as by the value of the contrast, that is defined as the ratio between the elastic moduli of the inclusions and the matrix [7, 8, 9].

25

The reliable evaluation of homogenized constitutive properties to be adopted for the macroscopic investigation of their behaviour pertains to the well-established homogenization theories, and is a long-standing problem of great interest for many researchers $10,11,12,13,14,15,16$, also with the purpose of designing innovative smart materials [17, 18. In this paper, we adopt a statistical ho- 
mogenization approach [11, 19] to derive the in-plane linear elastic constitutive properties polycristalline materials with thin layers interphases and to evaluate how they change in relation to contrast.

35 In the case of materials with random micro-structure, the lack of periodicity in the microscopic arrangement makes the homogenization process more challenging, asking for special attention in the detection of the Representative Volume Element (RVE) along with the evaluation of homogenized constitutive moduli. Several approaches have been proposed [20, 21, 22, 23, 24, 25, 26, 27, 14, 13,

40 28, 29, also referred to non-classical continua [30, 19, 9].

Among these models, we focus our attention on the possibility of approaching the RVE using finite-size scaling of intermediate control volume elements, named Statistical Volume Elements (SVEs), and proceed to homogenization (e.g. [31]). In this work we refer to [19], where a homogenization procedure 45 consistent with the Hill-Mandel condition 32] has been coupled with a statistical approach, by which scale-dependent bounds on classical moduli are obtained using Dirichlet and Neumann boundary conditions (BCs) for solving boundary value problems (BVPs). This statistically based homogenization procedure has provided significant results [30, 33, 34], with particular reference to the debated

50 problem of the convergence in the presence of materials with very low (or very high) contrast [9, 31, 25, 35.

More specifically, taking advantage of the encouraging results found in [36, 37, we here exploit an efficient evolution of the above mentioned procedure, i.e. the Fast Statistical Homogenization Procedure (FSHP) based on Virtual Element Method (VEM). In FSHP the statistical procedure is automatized and integrated in a completely in house specifically developed code, that makes it possible to quickly and efficiently perform a high number of parametric analyses. VEM [38, 39] is an innovative and very promising computational method that find many engineering and structural mechanics applications [40, 41, 42,

6o 41]: from linear [43, 44, 45] to non-linear elasticity 46, 47, 48, elastodynamic [49], topology optimization [50, 51], contact problem [52], plates [53], fracture 
and damage [54, 55], as well as in homogenization of heterogeneous materials [56, 57, 36, 37].

Here, the procedure is to specifically account for the microstructural topology 65 exhibited by polycrystalline composites with thin interphases, making the most of VEM to improve the computational efficiency. The result is a powerful numerical tool suitable for analyses of large portions of random microstrure, very important in the case materials with very high or low contrast [37. In particular the strategy proposed is to use a single virtual element for each grain (polygons of any shape with $\mathrm{n}$-nodes) with a significant reduction of the computational burden, while the interconnecting layer is discretized with a fine mesh of triangular elements. Other advantages of VEM are: capability of using hanging nodes, that permits local refinements and coupling different degree elements; robustness to distortion of the elements; perfectly coupling with FEM elements; accuracy because the stiffness matrix is computed in precision machine; easy implementation.

In this paper, low order virtual elements are used: the adoption of virtual elements of order one is particularly suitable for the homogenization procedure, as shown in [36, 37, 58. Moreover, stress/strain is constant over the elements, so but this approximation do not affect homogenization results. Furthermore, by the adoption of the hanging nodes we subdivide the edges of the grains for generating a sufficiently fine mesh in the interphase part.

Exploiting FSHP combined with VEM, several parametric analyses have be performed to characterize overall mechanical parameters of polycrystalline composites with thin interphase layers and to investigate their sensitivity to the contrast [59, 60]. Attention is paid both at the identification of the RVE size and at the evaluation of the overall mechanical properties, that vary in relation with the contrast of the material.

The outline of the paper is as follows. In Section 2 the statistical procedure эo is recalled and specialized to the case at hand of polycrystals with thin intergranular layers. Section 3 is devoted to the basic assumption of the first order virtual element formulation. Section 4 provides results of a set of parametric 
analyses on cermet-like polycrystals with intergranular phases stiffer of softer than grains. In order to check the capabilities of the proposed procedure, a successful comparison between the results obtained with VEM and those with a very fine FEM mesh is performed. In Section 5 some final remarks are presented, highlighting the expected advantages of the proposed approach with respect to more standard approaches. 


\section{Fast Statistical Homogenization Procedure}

high contrast materials [19].

In order to perform homogenization, we describe the material at two scales of interest: the microscopic and the macroscopic levels. At the microscopic level the 


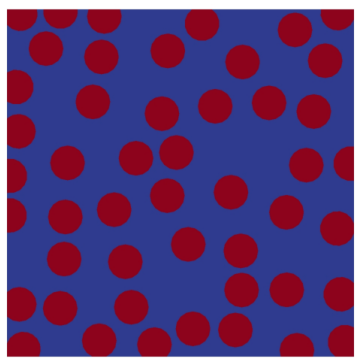

(a) Two-phases material with circular inclusions

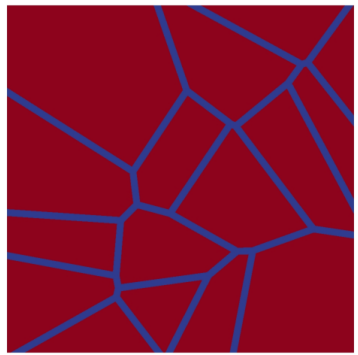

(b) Polycrystal material with thin interfaces

Figure 1: Portions of heterogeneous material. The matrix and inclusions are depicted in red and blue, respectively.

heterogeneous material is represented in detail, accounting for each constituent in terms of geometry and constitutive behaviour. At the macroscopic level the composite material is ideally replaced by an equivalent material whose global behaviour is representative of the actual heterogeneous material. The governing equations are formally the same as those defined at the microscopic level, except for the constitutive law that is not 'a priori' defined at the macroscopic level, but directly descends from the lower level as result of the homogenization procedure. In the following, lower case letters always refer to the micro-scale, while upper case letters to the macro-scale.

We refer to a linearised two-dimensional framework. At the lower level each material phase is characterized by linear elastic isotropic behaviour with the 
stress-strain relations written as:

$$
\sigma_{i j}=\lambda \varepsilon_{k k} \delta_{i j}+2 \mu \varepsilon_{i j}
$$

where $\varepsilon_{i j}$ and $\sigma_{i j}(i, j, k=1,2)$ are the components of the micro-strain and micro-stress tensors, $\lambda$ and $\mu$ are the Lamé constants, and $\delta_{i j}(i, j=1,2)$ is the Kronecker symbol. At the macroscopic level, the general anisotropic stressstrain relations, read:

$$
\Sigma_{i j}=\mathbb{C}_{i j h k} E_{h k}
$$

where $E_{i j}, \Sigma_{i j}(i, j=1,2)$ are the components of the macro-strain and macrostress tensors and $\mathbb{C}_{i j h k}(i, j, h, k=1,2)$ are the homogenized moduli, i.e the components of the macroscopic elastic tensor obtained via a homogenization procedure based on the Hill's macro-homogeneity condition [32]:

$$
\Sigma_{i j} E_{i j}=\frac{1}{A_{\delta}} \int_{\mathcal{B}_{\delta}} \sigma_{i j} \varepsilon_{i j} d A
$$

which states an energy equivalence between the macroscopic point and the corresponding domain (test window), $\mathcal{B}_{\delta}$, occupying a region of area $A_{\delta}$ at the microscopic level.

The homogenization procedure is based on the solution of properly defined boundary value problems at the microscopic level with Dirichlet and Neumann boundary conditions on the boundary $\partial \mathcal{B}_{\delta}$, directly deriving from the fulfilment of the macro-homogeneity condition, Eq. (3). The Dirichlet and Neumann BCs reads, respectively:

$$
\underbrace{u_{i}=E_{i j} x_{j}, \text { on } \partial \mathcal{B}_{\delta}}_{\text {Dirichlet BCs }}, \quad \underbrace{t_{i}=\Sigma_{i j} n_{j}, \text { on } \partial \mathcal{B}_{\delta}}_{\text {Neumann } B C s},
$$

$u_{i}$ being the components of the displacement vector and $x_{j}(i, j=1,2)$ the coordinates of the generic point on the boundary, $\partial \mathcal{B}_{\delta}$, with respect to a reference system with origin in the geometric center of the test window $\mathcal{B}_{\delta} . t_{i}$ are the components of the traction vector and $n_{j}$ the component of the outward normal to $\partial \mathcal{B}_{\delta}(i, j=1,2)$. 
FSHP is based on the statistical homogenization procedure previously developed in [19] and briefly described in Section 1. The proposed homogenization procedure is conceived for evaluating both the homogenized elastic parameters of a non-periodic heterogeneous material and for identify the Representative

140 Volume Element (RVE), that in the absence of a repetitive micro-structure is not known a priori.

According to the approach presented in [19], as well as in [21, 11, the presented procedure requires the statistical definition of a number of realizations called Statistical Volume Elements (SVEs), representing the micro-structure, sampled in a Monte Carlo sense, which allows for determining series of scaledependent upper and lower bounds for the overall elastic moduli and to approach the RVE size, $\delta_{R V E}$, using a statistical stopping criterion, which is based on the variation of the average elastic moduli.

All steps of the homogenization procedure are completely integrated in the FSHP and they are described below.

Step 1 Input: in the case of disk-shaped inclusion, we set set the nominal volume fraction of the medium $(\rho \leq 40 \%)$ as $\sum_{k=1}^{n^{i}} A_{k} / L^{2}$ in which $A_{k}$ and $L^{2}$ are the area of $k$-th inclusion and area of a test window, respectively. If the particles are disk-shaped inclusions, with diameter $d$, randomly distributed on the base of a planar 'hard-core' Poisson point field with distance, $s$, between the particles, fixed in order to avoid very narrow necks between inclusions. Define the dimensionless scale factor $\delta=L / d$. In the case of polycrystalline materials with thin interfaces, we set the average dimension of the grains $\bar{d}$ and define the dimensionless scale factor $\delta=L / \bar{d}$. The parameter $s$ is the thickness of the layer around the grains and in this work it is assumed constant for all grains boundaries.

Fix the mechanical parameters of each phase: Young modulus of inclusions and matrix, $E_{i}$ and $E_{m}$; Poisson coefficient, $\nu_{i}$ and $\nu_{m}$. Set the minimum number of simulations for convergence, $N^{l i m}$, and a tolerance parameter, 
Step 2 Input: initialize the window size, $L=L_{0}$, and number of simulations, $N=N_{0}$.

Step 3 Realizations: for the case of circular inclusion the procedure automatically determine for each window size the number of inclusions (Poisson random variable) via simulations exploit Knuth's algorithm (see [59, 36]). Then simulate random dispositions of disks' centers, according to the uniform distribution, that are the realizations (SVEs) of portions of the random medium. Otherwise, the procedure generate a random polygonal mesh with average dimension of grains $\bar{d}$ calling the Matlab ${ }^{\circledR}$ program PolyMesher developed by [62. Then all edges are shifted inwards by $s / 2$ in order to obtain the final geometry of all realizations $\mathcal{B}_{\delta}$. In Fig. 2 a schematic of the procedure is depicted for a generic grain: the initial polygon has vertices $P_{i}$; additional points $\hat{P}_{i}$ are identified as a result of the shifting procedure, so that the interface area (in light blue) and the grain area (in light red) are defined.

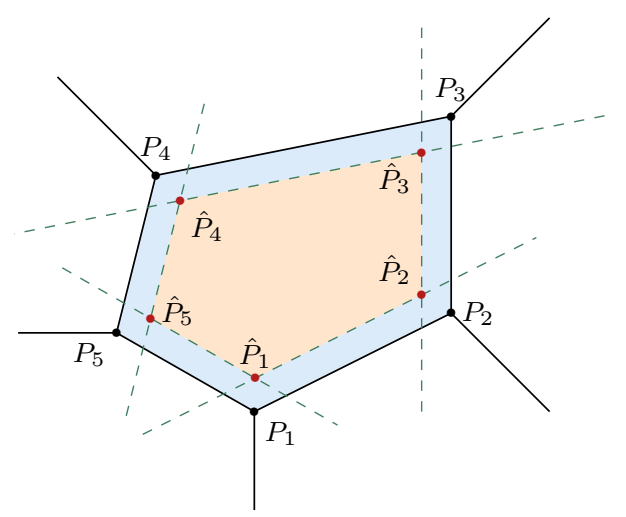

Figure 2: Example of shift procedure starting by initial polygonal mesh obtained by PolyMesher 62

In all cases, each realization is supposed to be independent from any previ- 


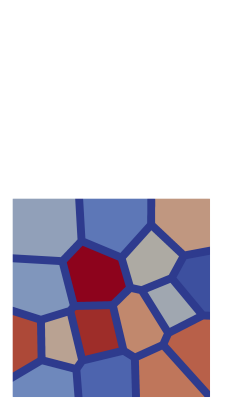

(a) $L=100 \mu m$

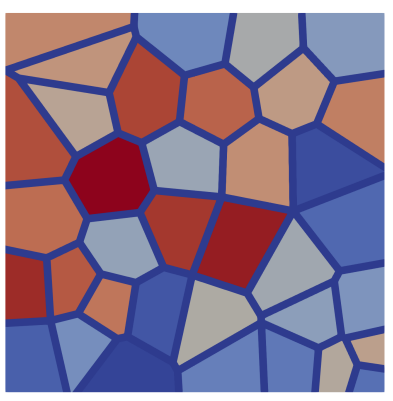

(b) $L=200 \mu m$

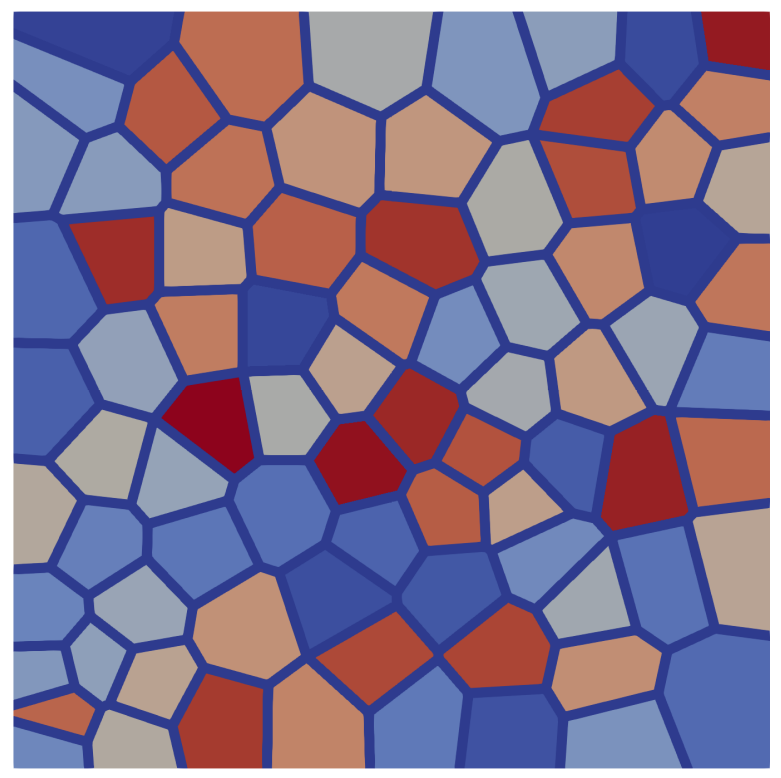

(c) $L=300 \mu m$

Figure 3: Example of realization obtained by FSHP for different window size $L$

ous one. An examples of realization of the micro-structure and the related generated mesh for circular and polycrystal inclusions are shown in Figs. 1(a) 1(b) In Fig. 3 a realization obtained by FSHP for different windows size has been plotted.

Step 4 Generate/Solve: for each SVE, generate the relative mesh and solve both the Dirichlet and Neumann (Eq. (4)) BVP, and compute the homogenized 
constitutive parameters.

Step 5 Compute: evaluate the average bulk modulus, $\langle\mathbb{K}\rangle_{\delta}$, the relative standard deviation $\sigma\left(\langle\mathbb{K}\rangle_{\delta}\right)$ and variation coefficient $C V\left(\langle\mathbb{K}\rangle_{\delta}\right)$. Then compute $N_{i}=\left(1.96 \sigma\left(\langle\mathbb{K}\rangle_{\delta}\right) /\left(\langle\mathbb{K}\rangle_{\delta} T o l\right)^{2}\right.$, which ensures that the confidence interval of the average homogenized constitutive parameter set at $95 \%$, evaluated over the normal standard distribution, is within the tolerance allowed, Tol. Repeat Steps 3-4 until $N_{i}<N^{l i m}$.

195 Step 6 Checking: if the number of realizations necessary for ensuring the requirement at Step 5 is small enough, stop the procedure. We choose as the number of realizations necessary the most unfavourable number between those obtained by solving BVPs of Neumann or Dirichlet. Otherwise choose an increased value of $\delta$ and go to Step 3 .

200

The fulfilment of the requirement at Step 6 means that the values of the homogenized constitutive coefficients are distributed around their averages with a vanishing variation coefficient, and that the RVE size is achieved. The effective homogenized elastic moduli can be determined as the mean value between 205 (RVE).

The statistical convergence criterion adopted is based on a $95 \%$ confidence level of the Normal Standard distribution, which provides the number $N$ of realizations at which is possible to stop the simulations for a given window size $\delta$.

210 When this number is small enough, the average values of the effective moduli converge and the RVE size is achieved. This circumstance also corresponds to reaching the minimum window size $\delta_{R V E}$ for which the estimated homogenized moduli remain constant, within a tolerance interval less than $0.5 \%$ for both the Dirichlet and Neumann solutions. The minimum number of simulations, $N^{\text {lim }}$, 215 and the tolerance parameter, Tol, are chosen in order to define a narrow confidence interval for the average and to obtain a reliable convergence criterion. The adopted statistical criterion allows us to detect the RVE size also when the 
Dirichlet and Neumann solutions do not tend to the same value. The values of the tolerance are assumed as a function of the data dispersion [19].

220 For all realizations of the micro-structure the Virtual Element Method (VEM) has been adopted as a numeric tool, that permits to adopt single polygonal element for the inclusions without meshing with consequently high reduction of the computational burden with respect to finite elements. Moreover, the adoption of the VEM allows us to create a refined mesh in the interface without meshing the granular elements thanks to the use of so-called hanging nodes (Fig. 5). In the zones of interfaces a triangular virtual element mesh have been adopted, determined using a code for generating random mesh of Delaunay type (Triangle, 63]). As demonstrated in [64, three nodes virtual elements behaves just like 3-noded triangular finite elements.

230 The computational strategies adopted are aimed at making the statistical homogenization process as efficient as possible. This allow us to fast solve series (hundreds) of BVPs and to rapidly converge to the RVE solution. 


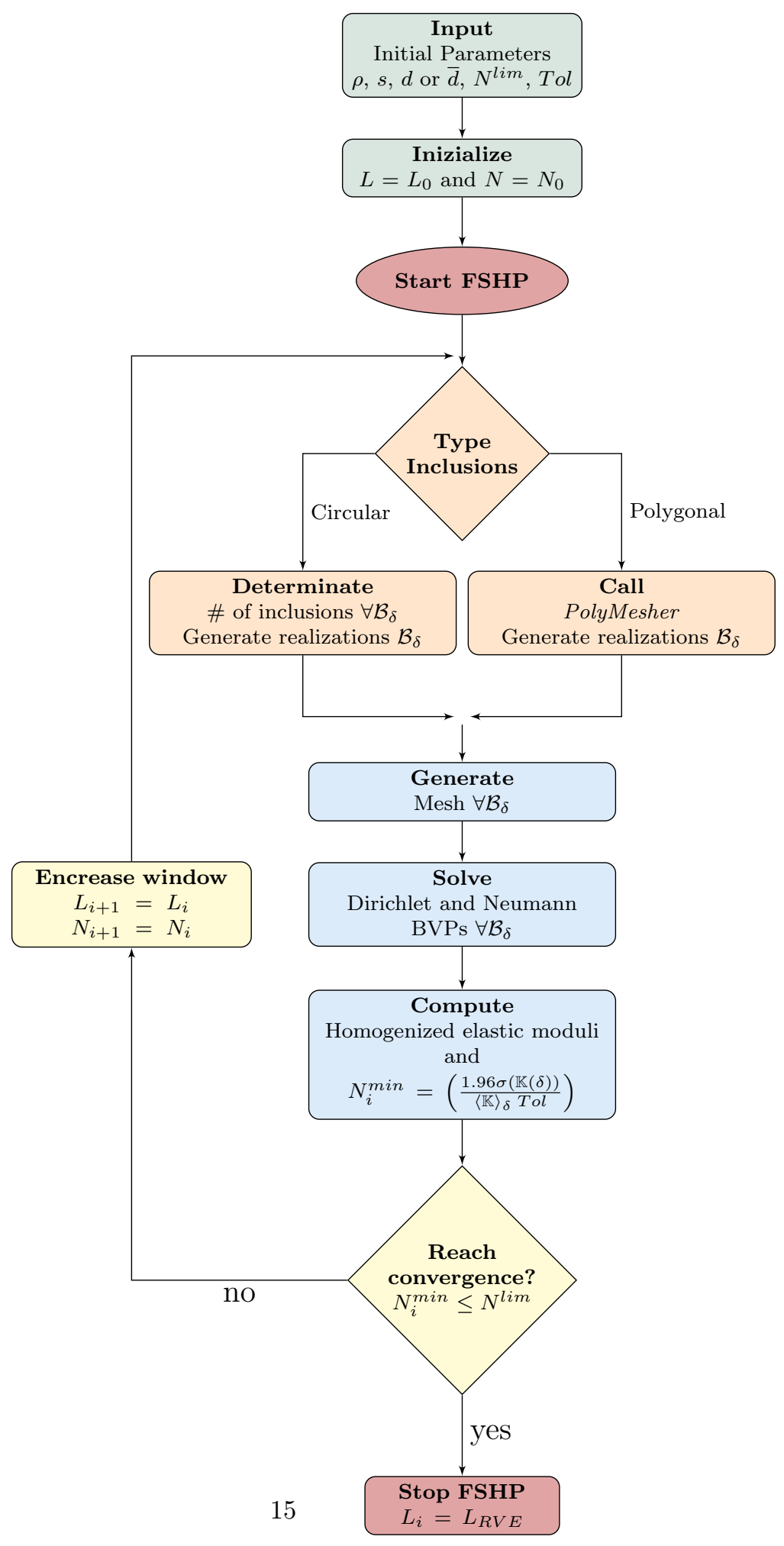

Figure 4: Flow-chart of the Fast Statistical Homogenization Procedure 


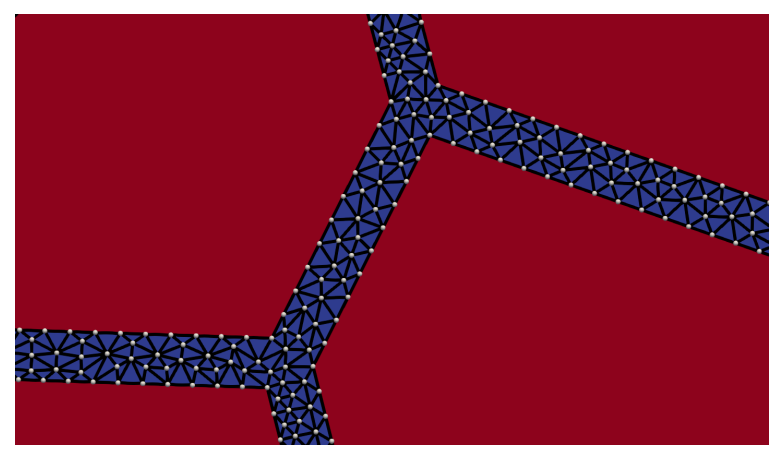

Figure 5: Example of the mesh in the interface zone between the grains obtained using the procedure 


\section{Virtual Element Framework}

In this section we briefly recall the governing equation of the linear elastostatic

problem and the related weak formulation, mandatory starting point to describe the construction of virtual element. We restrict our investigation to virtual elements of degree 1 [38, 39] also referred as lower order virtual elements. The Voigt notation is adopted since is more suited to easily develop the VEM formulation.

\subsubsection{The linear elastic problem}

240 We consider a two-dimensional domain, $\Omega$, with $\Gamma$ being its boundary. The body is subjected to the volume force, represented by the vector $\boldsymbol{f} \in\left(L^{2}(\Omega)\right)^{2}$, $\boldsymbol{f}=\left\{f_{1}, f_{2}\right\}^{T}$ (within the standard Lebesgue space), and given boundary conditions, under the hypothesis of small deformations. For the sake of simplicity, we consider homogeneous Dirichlet boundary conditions to define the deformation problem of a linear elastic continuum. We introduce the Sobolev space, $\boldsymbol{V}:=\left(H_{0}^{1}(\Omega)\right)^{2}$, of the admissible displacement fields, represented by the vector $\boldsymbol{v}$. Furthermore we represent the strain as the vector $\varepsilon(\boldsymbol{u})$ associated to the displacement field vector $\boldsymbol{u}=\left\{u_{1}, u_{2}\right\}^{T}$ :

$$
\boldsymbol{\varepsilon}(\boldsymbol{u})=\left\{\varepsilon_{11}, \varepsilon_{22}, 2 \varepsilon_{12}\right\}^{T}=\left\{\partial_{x} u_{1}, \partial_{y} u_{2}, \partial_{y} u_{1}+\partial_{x} u_{2}\right\}^{T},
$$

where $\partial_{(\cdot)}$ denotes the partial derivative with respect to the $(\cdot)$-coordinate.

The weak form of the linear elastostatic problem, provided by the virtual work principle, reads:

$$
\left\{\begin{array}{l}
\text { Find } \boldsymbol{u} \in \boldsymbol{V} \text { such that : } \\
a(\boldsymbol{u}, \boldsymbol{v})=\mathcal{F}(\boldsymbol{v}) \quad \forall \boldsymbol{v} \in \boldsymbol{V}
\end{array}\right.
$$
the real numbers, reads:

$$
a(\boldsymbol{u}, \boldsymbol{v})=\int_{\Omega} \boldsymbol{\varepsilon}(\boldsymbol{v})^{T} \mathbb{C} \varepsilon(\boldsymbol{u}) d \Omega
$$


in witch $\mathbb{C}$ is the $3 \times 3$ elastic tensor, and the linear functional $\mathcal{F}(\cdot): \boldsymbol{V} \rightarrow \mathbb{R}$, reads:

$$
\mathcal{F}(\boldsymbol{v})=\int_{\Omega} \boldsymbol{f}^{T} \boldsymbol{v} d \Omega
$$

\subsubsection{Virtual element formulation}

In this subsection we introduce the virtual element discretization used in the homogenization procedure. In order to approximate the solution of the problem (6) we consider a decomposition $\mathcal{T}_{h}$ of the domain $\Omega$ into non overlapping polygonal elements $E$. In the following, we denote by $e$ the straight edges of the mesh $\mathcal{T}_{h}$ and, for all $e \in \partial E, \boldsymbol{n}_{i}$ denotes the outward unit normal vector to $e_{i}$ (Fig. 6(a) . The symbol $n_{e}$ represents the number of the edges of the polygon E.

Let $k$ be an integer $\geq 1$. Let us denote by $\mathcal{P}_{k}(\Omega)$ the space of polynomials, living on the set $\Omega \subseteq \mathbb{R}^{2}$, of degree less than or equal to $k$.

By the discretization introduced, it is possible to write the bilinear form (6), as in the finite element methodology, in the following way:

$$
a(\boldsymbol{u}, \boldsymbol{v})=\sum_{E \in \mathcal{T}_{h}} a^{E}(\boldsymbol{u}, \boldsymbol{v}) \quad \forall \boldsymbol{v} \in \boldsymbol{V}
$$

The discrete virtual element space, $\boldsymbol{V}_{h}$, is:

$$
\boldsymbol{V}_{h}:=\left\{\boldsymbol{v} \in \boldsymbol{V}:\left.\boldsymbol{v}\right|_{E} \in \boldsymbol{V}_{h \mid E} \forall E \in \mathcal{T}_{h}\right\}
$$

where the local space, $\boldsymbol{V}_{h \mid E}:=\left[V_{h \mid E}\right]^{2}$. For the virtual element of degree $k=1$, $V_{h \mid E}$ is defined as:

$$
V_{h \mid E}:=\left\{\boldsymbol{v}_{h} \in H^{1}(E) \cap C^{0}(E): \triangle \boldsymbol{v}_{h} \in\{0\},\left.\boldsymbol{v}_{h}\right|_{e} \in \mathcal{P}_{1}(e) \forall e \in \partial E\right\} .
$$

The dimension of the space $\boldsymbol{V}_{h \mid E}$ then is:

$$
m=\operatorname{dim}\left(\boldsymbol{V}_{h \mid E}\right)=2 n_{e} .
$$

We can observe that, in contrast to the standard finite element approach, the local space $\boldsymbol{V}_{h \mid E}$ is not fully explicit. Moreover $\boldsymbol{v}_{h}$ is a polynomial of degree 1 on each edge $e$ of $E$ and globally continuous on $\partial E$. 


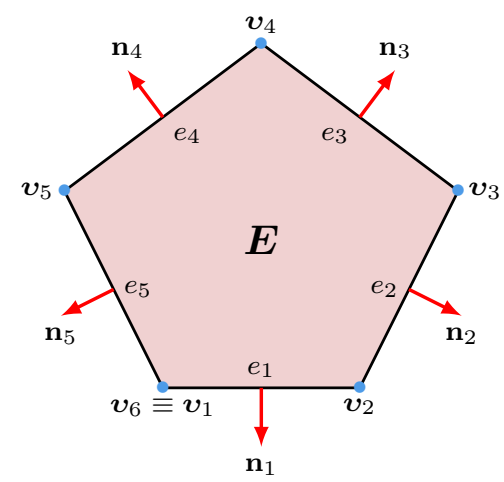

(a)

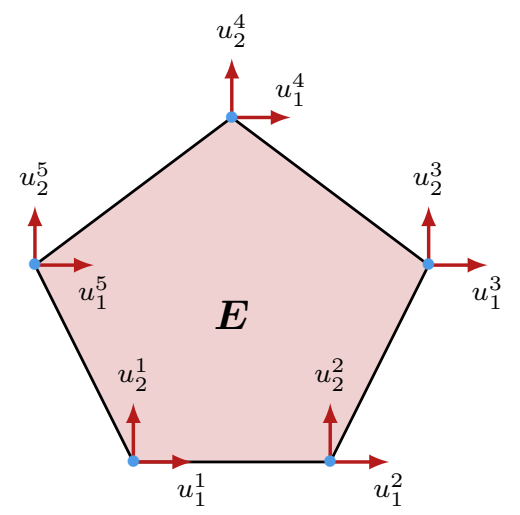

(b)

Figure 6: Example of virtual element, $E$, with the relative nodes and edges numeration (a) and degrees of freedom of the virtual element of degree 1 (b).

The problem (6) restricted to the discrete space $\boldsymbol{V}_{h}$ becomes:

$$
\left\{\begin{array}{l}
\text { Find } \boldsymbol{u}_{h} \in \boldsymbol{V}_{\boldsymbol{h}} \text { such that } \\
a_{h}\left(\boldsymbol{u}_{h}, \boldsymbol{v}_{h}\right)=\mathcal{F}\left(\boldsymbol{v}_{h}\right) \quad \forall \boldsymbol{v}_{h} \in \boldsymbol{V}_{\boldsymbol{h}},
\end{array}\right.
$$

where $a_{h}(\cdot, \cdot): \boldsymbol{V}_{h} \times \boldsymbol{V}_{h} \rightarrow \mathbb{R}$ is the discrete bilinear form approximating the continuous form $a(\cdot, \cdot)$ and, $\mathcal{F}\left(\boldsymbol{v}_{h}\right)$ is the term approximating the virtual work of external load. In this work the body force is null, so no details for its imple- 
mentation are reported. The discrete bilinear form is constructed element by element as

$$
a_{h}\left(\boldsymbol{u}_{h}, \boldsymbol{v}_{h}\right)=\sum_{E \in \mathcal{T}_{h}} a_{h}^{E}\left(\boldsymbol{u}_{h}, \boldsymbol{v}_{h}\right) \quad \forall \boldsymbol{u}_{h}, \boldsymbol{v}_{h} \in \boldsymbol{V}_{h},
$$

265 vations can be made:

- the functions $\boldsymbol{v}_{h} \in \boldsymbol{V}_{h \mid E}$ are explicitly known on $\partial E$ (linear functions);

- the functions $\boldsymbol{v}_{h} \in \boldsymbol{V}_{h \mid E}$ are not explicitly known inside the element $E$;

- $\left(\mathcal{P}_{1}(E)\right)^{2} \subseteq \boldsymbol{V}_{h \mid E}$.

270 $\boldsymbol{v}_{h}\left(v_{i}\right) i=1,2, \ldots, n_{e}$, where $v_{i}$ is the $i$-th corner of $E$.

\subsubsection{Projection operator and construction of the stiffness matrix}

In accordance to [65] we define the projector operator of the strain as:

$$
\begin{aligned}
& \Pi: \boldsymbol{V}_{h \mid E} \rightarrow \mathcal{P}_{0}(E)_{\text {sym }}^{2 \times 2} \\
& \boldsymbol{v}_{h} \rightarrow \Pi\left(\boldsymbol{v}_{h}\right)
\end{aligned}
$$

More specifically, the projector operator respects the following orthogonality condition:

$$
\int_{E} \Pi\left(\boldsymbol{v}_{h}\right)^{T} \hat{\varepsilon} d E=\int_{E} \varepsilon\left(\boldsymbol{v}_{h}\right)^{T} \hat{\varepsilon} d E \quad \forall \hat{\varepsilon} \in \mathcal{P}_{0}(E)_{s y m}^{2 \times 2} .
$$

As in the finite element method, we define the vectors $\boldsymbol{v}_{h} \in \boldsymbol{V}_{h \mid E}$ and $\hat{\varepsilon} \in$ $\mathcal{P}_{0}(E)_{\text {sym }}^{2 \times 2}$ using the degrees of freedom $\overline{\boldsymbol{v}}_{h} \in \mathbb{R}^{2 n_{e}}, \overline{\boldsymbol{\varepsilon}} \in \mathbb{R}^{3}$ :

$$
\left\{\begin{array}{l}
\boldsymbol{v}_{h}=\boldsymbol{N}^{V} \overline{\boldsymbol{v}}_{h}, \\
\hat{\boldsymbol{\varepsilon}}=\boldsymbol{N}^{u} \overline{\boldsymbol{\varepsilon}} .
\end{array}\right.
$$

The matrices $\boldsymbol{N}^{u}$ and $\boldsymbol{N}^{V}$ are

$$
\boldsymbol{N}^{u}=\left[\begin{array}{ccc}
1 & 0 & 0 \\
0 & 1 & 0 \\
0 & 0 & 1
\end{array}\right], \quad \boldsymbol{N}^{V}=\left[\begin{array}{ccccc}
\phi_{1} & 0 & \cdots & \phi_{n_{e}} & 0 \\
0 & \phi_{1} & \cdots & 0 & \phi_{n_{e}}
\end{array}\right]
$$


where $\phi_{i}$ is the standard $i-$ th shape function. The projection operator in matrix is:

$$
\Pi\left(\boldsymbol{v}_{h}\right)=\boldsymbol{N}^{u} \bar{\Pi} \overline{\boldsymbol{v}}_{h}
$$

with $\bar{\Pi} \in \mathbb{R}^{3 \times m}$.

By putting Eqs. (17), 19p into the Eq. 116) we obtain:

$$
\int_{E}\left(\boldsymbol{N}^{u} \bar{\Pi} \overline{\boldsymbol{v}}_{h}\right)^{T} \boldsymbol{N}^{u} \overline{\boldsymbol{\varepsilon}} d E=\int_{E}\left[\varepsilon\left(\boldsymbol{N}^{V} \overline{\boldsymbol{v}}_{h}\right)\right]^{T} \boldsymbol{N}^{u} \overline{\boldsymbol{\varepsilon}} d E \quad \forall \overline{\boldsymbol{\varepsilon}} \in \mathbb{R}^{3} .
$$

Integrating by part the right hand side we obtain:

$$
\int_{E}\left(\boldsymbol{N}^{u} \bar{\Pi} \overline{\boldsymbol{v}}_{h}\right)^{T} \boldsymbol{N}^{u} \overline{\boldsymbol{\varepsilon}} d E=\int_{\partial E}\left(\boldsymbol{N}^{V} \overline{\boldsymbol{v}}_{h}\right)^{T}\left(\boldsymbol{N}_{E} \boldsymbol{N}^{u} \overline{\boldsymbol{\varepsilon}}\right) d \partial E \quad \forall \overline{\boldsymbol{\varepsilon}} \in \mathbb{R}^{3},
$$

where $\boldsymbol{N}_{E}$ contains the components of the outward normal $\boldsymbol{n}=\left\{n_{1}, n_{2}\right\}^{T}$, i.e.

$$
\boldsymbol{N}_{E}=\left[\begin{array}{ccc}
n_{1} & 0 & n_{2} \\
0 & n_{2} & n_{1}
\end{array}\right] \text {. }
$$

After some algebra:

$$
\overline{\boldsymbol{\varepsilon}}^{T}\left\{\int_{E}\left(\boldsymbol{N}^{u}\right)^{T} \boldsymbol{N}^{u} d E\right\} \bar{\Pi} \overline{\boldsymbol{v}}_{h}=\overline{\boldsymbol{\varepsilon}}^{T}\left\{\int_{\partial E}\left(\boldsymbol{N}_{E} \boldsymbol{N}^{u}\right)^{T} \boldsymbol{N}^{V} d \partial E\right\} \overline{\boldsymbol{v}}_{h} \quad \forall \overline{\boldsymbol{\varepsilon}} \in \mathbb{R}^{3},
$$

Finally the local projector operator $\bar{\Pi}$ is:

$$
\bar{\Pi}=\boldsymbol{G}^{-1} \boldsymbol{B},
$$

where:

$$
\boldsymbol{G}=\int_{E}\left(\boldsymbol{N}^{u}\right)^{T} \boldsymbol{N}^{u} d E, \quad \boldsymbol{B}=\int_{\partial E}\left(\boldsymbol{N}_{E} \boldsymbol{N}^{u}\right)^{T} \boldsymbol{N}^{V} d \partial E,
$$

We can observe that the matrix $G \in \mathbb{R}^{3 \times 3}$ is computed by knowing the area of the elements $|E|$ :

$$
\boldsymbol{G}=\left[\begin{array}{ccc}
|E| & 0 & 0 \\
0 & |E| & 0 \\
0 & 0 & |E|
\end{array}\right]
$$

where $|E|$ is computed using the Gauss-Green formula:

$$
|E|=\iint_{E} d x d y=\int_{\partial E^{+}} x d y
$$


where $\partial E^{+}$is the boundary of the element oriented anticlockwise and the matrix $\boldsymbol{B} \in \mathbb{R}^{3 \times m}:$

$$
\boldsymbol{B}=\left[\begin{array}{ccccc}
\int_{\partial E} n_{1} \phi_{1} d \partial E & 0 & \cdots & \int_{\partial E} n_{1} \phi_{n_{e}} d \partial E & 0 \\
0 & \int_{\partial E} n_{2} \phi_{1} d \partial E & \cdots & 0 & \int_{\partial E} n_{2} \phi_{n_{e}} d \partial E \\
\int_{\partial E} n_{2} \phi_{1} d \partial E & \int_{\partial E} n_{1} \phi_{1} d \partial E & \cdots & \int_{\partial E} n_{2} \phi_{n_{e}} d \partial E & \int_{\partial E} n_{1} \phi_{n_{e}} d \partial E
\end{array}\right]
$$

where the boundary integrals in the matrix $\boldsymbol{B}$ are computed using the degrees of freedom of the element. In particular, the boundary integrals are computed resorting to the Gauss-Lobatto quadrature rule with $k+1$ points, since this choice has turned to be adequate. In the case of VEM $k=1$ we can obtain:

$$
\begin{aligned}
& \int_{\partial E} n_{1} \phi_{i} d \partial E=\frac{1}{2}\left(n_{1}^{i-1} l_{i-1}+n_{1}^{i} l_{i}\right), \quad \text { for } i=1, \cdots, n_{e} \\
& \int_{\partial E} n_{2} \phi_{i} d \partial E=\frac{1}{2}\left(n_{2}^{i-1} l_{i-1}+n_{2}^{i} l_{i}\right),
\end{aligned}
$$

where $l_{i}=\left|e_{i}\right|$ is the length of the $i$-th edge of the element $E$ and $n_{j}^{i}(i=$ $\left.2751, \cdots, n_{e} ; j=1,2\right)$ is the $i$-th outward normal of components $j$. Moreover if $i-1=0$ use $i=n_{e}$ instead to $i=0$.

The main difference between Finite Element Method and Virtual Element Method regards the approximation of the bilinear form of Eq. 114, that for the VEM is as follow

$$
a_{h}^{E}\left(\boldsymbol{u}_{h}, \boldsymbol{v}_{h}\right)=\underbrace{\int_{E}\left[\Pi\left(\boldsymbol{v}_{h}\right)\right]^{T} \mathbb{C} \Pi\left(\boldsymbol{u}_{h}\right) d E}_{\overline{\boldsymbol{v}}_{h}^{T} \boldsymbol{M} \overline{\boldsymbol{u}}_{h}:=\text { consistency term }}+\underbrace{S^{E}\left(\boldsymbol{u}_{h}, \boldsymbol{v}_{h}\right)}_{\overline{\boldsymbol{v}}_{h}^{T} \boldsymbol{K}_{s} \overline{\boldsymbol{u}}_{h}:=\text { stability }},
$$

where the first term of the right hand side is the consistent term and the second term is the stabilization term. The stabilization part is not presented in Finite Element Method and it is one of the peculiarity of the VEM. For computing the consistent term $\boldsymbol{M}$ we use Eq. 19 and Eq. (7)

$$
\int_{E}\left[\Pi\left(\boldsymbol{v}_{h}\right)\right]^{T} \mathbb{C} \Pi\left(\boldsymbol{u}_{h}\right) d E=\overline{\boldsymbol{v}}_{h}^{T} \bar{\Pi}^{T}\left[\int_{E}\left(\boldsymbol{N}^{u}\right)^{T} \mathbb{C} \boldsymbol{N}^{u} d E\right] \bar{\Pi} \overline{\boldsymbol{u}}_{h},
$$

where the stiffness matrix $\boldsymbol{M}$ is defined as

$$
\boldsymbol{M}=\bar{\Pi}^{T}\left[\int_{E}\left(\boldsymbol{N}^{u}\right)^{T} \mathbb{C} \boldsymbol{N}^{u} d E\right] \bar{\Pi} .
$$


Eq. 32 has been rearranged in compact form as follow adopting a constant elastic tensor

$$
M=\bar{\Pi}^{T} \mathbb{C} G \bar{\Pi} .
$$

Concerning the stabilization term, we adopt the choice introduced in 38, 39. However other authors have proposed a different stabilization terms [66]. Some preliminaries are required before introducing the stabilization term. In particular we introduce the space of the scaled monomials $\boldsymbol{p} \in\left(\mathcal{P}_{k}(E)\right)^{2}$ since $\left(\mathcal{P}_{k}(E) \subset \boldsymbol{V}_{h}(E)\right)^{2}$. In the case of VEM of degree $k=1$ the space of the scaled monomials is

$$
\left(\mathcal{P}_{k}(E)\right)^{2}=\operatorname{span}\left\{\left(\begin{array}{l}
1 \\
0
\end{array}\right),\left(\begin{array}{l}
0 \\
1
\end{array}\right),\left(\begin{array}{l}
\xi \\
0
\end{array}\right),\left(\begin{array}{l}
0 \\
\xi
\end{array}\right),\left(\begin{array}{l}
\eta \\
0
\end{array}\right),\left(\begin{array}{l}
0 \\
\eta
\end{array}\right)\right\},
$$

where $\xi=\left(x-x_{E}\right) / h_{E}, \eta=\left(y-y_{E}\right) / h_{E},\left(x_{E}, y_{E}\right)$ are the coordinates of the centre of $E$ and $h_{E}$ its diameter. The stabilization term is defined as follow

$$
\boldsymbol{K}_{s}=\tau \operatorname{tr}(\boldsymbol{M})\left[\boldsymbol{I}-\boldsymbol{D}\left(\boldsymbol{D}^{T} \boldsymbol{D}\right)^{-1} \boldsymbol{D}^{T}\right],
$$

where $\tau \in \mathbb{R}$ is a coefficient defined by the user that can be set equal to $1 / 2$ for linear elasticity [65] and $\operatorname{tr}(\cdot)$ denotes the trace operator. The matrix $\boldsymbol{D} \in \mathbb{R}^{m \times 6}$ collects the values of the polynomials $\boldsymbol{p}_{i}$ at the degrees of freedom on $E$ (nodes of the element in the case of degree $k=1$ ). An accurate inspection of Eq. (35) reveals that the stability term is basically a rough approximation of the internal energy associated with the difference between VEM shape function and its projection, while $\tau \operatorname{tr}(\boldsymbol{M})$ scales the term with respect to the consistency part, which is the properly used for providing convergence of the method [38].

\subsubsection{Local load vector}

Regarding the approximation of right hand side of the equation $(13)$, the same assumption proposed in 65 is used, i.e.

$$
\mathcal{F}\left(\boldsymbol{v}_{h}\right)=\left(\int_{E} \boldsymbol{f} d E\right)^{T} \sum_{i=1}^{n_{e}} \frac{1}{n_{e}} \boldsymbol{v}_{h}\left(v_{i}\right),
$$

so that the body load over the element $E$ is subdivided over all nodes $v_{i}$ of the polygonal element. 


\section{Numerical Results}

In this section, the results of parametric analyses performed using the described computational FSHP in conjunction with VEM are provided. We restrict our investigation to polycrystalline material with thin interfaces, since the other type of composite has been already extensively studied in [67, 58, 68,

First, the reliability of the assumptions for the mesh topology, i.e. using one virtual element to model the grain and several triangular virtual elements to model the thin interfaces, is investigated by comparing the results with those obtained via a very dense triangular finite element mesh. Two distinct cases are studied: material A with stiff grains in softer interfaces and, material B with soft grains in stiffer interfaces. The analyses are performed for different level of material contrasts, $c$, between the Young moduli of constituents (inclusions/matrix). In the case of polycristal materials the grains and interface layers play the role of inclusions and matrix, respectively.

\subsection{Comparison between VEM and FEM discretization}

As a preliminary test, we consider an ideal material that mimics a cermet-like composite with grains and thin interfaces and investigate, for a given window size, the results obtained via VEM and FEM discretizations. We consider the window size $L=100 \mu \mathrm{m}$, the average dimension of grains and the thickness interfaces equal to $\bar{d}=40 \mu \mathrm{m}$ and $s=2 \mu \mathrm{m}$, respectively. The elastic moduli of the interface layer are $E_{m}=1.0 \mathrm{MPa}$ and $\nu_{m}=0.25$, while for the grains $E_{i}=1,2, \ldots, 10 M P a$ and $\nu_{m}=0.25$.

The components of the homogenized elastic tensor $\overline{\mathbb{C}}_{i j h k}$ are evaluated using both Dirichlet (apex D) and Neumann (apex N) boundary conditions. The structure of the computed homogenized stiffness matrix is equal to:

$$
\overline{\mathbb{C}}=\left[\begin{array}{ccc}
\overline{\mathbb{C}}_{1111} & \overline{\mathbb{C}}_{1122} & 0 \\
\overline{\mathbb{C}}_{2211} & \overline{\mathbb{C}}_{2222} & 0 \\
0 & 0 & \overline{\mathbb{C}}_{1212}
\end{array}\right]
$$


More specifically, the results obtained using with virtual elements are compared to those obtained using finite elements, see Fig $7(\mathrm{~b})$ for details on the mesh used. The FEM mesh adopted for the simulation has 15749 \#Nodes, while the VEM mesh has 3493 \#Nodes.

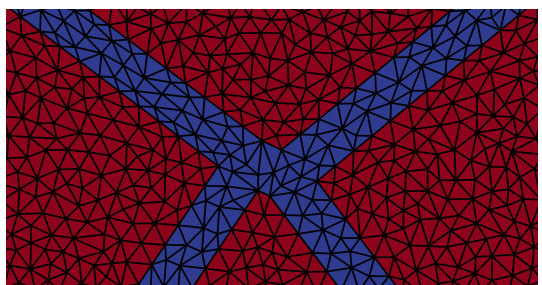

(a) FEM mesh

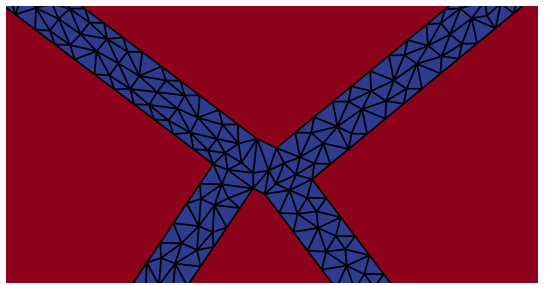

(b) VEM mesh

Figure 7: Example of the meshes adopted: FEM mesh (a) and VEM mesh (b)

In Tabs. 1 and 2 the homogenized moduli obtained for different level of

315 contrast have been reported $\left(1 \leq E_{i} / E_{m} \leq 10\right)$ using both Dirichlet and Neumann boundary conditions, respectively. The solution obtained adopting the VEM mesh is always close to the reference solution FEM. The maximum errors, computed as $e=\left(\overline{\mathbb{C}}_{i j h k}^{F E M}-\overline{\mathbb{C}}_{i j h k}^{V E M}\right) / \overline{\mathbb{C}}_{i j h k}^{F E M}$, in the case of the highest contrast considered, $E_{i} / E_{m}=10$, are $0,79 \%$ and $0.17 \%$ for $\overline{\mathbb{C}}_{2222}^{D}$ and $\overline{\mathbb{C}}_{1111}^{N}$, respectively.

320 The obtained results highlight the high performance of the VEM methodology in these applications. VEM preserves $\sim 300 \%$ of the degrees of freedom respect to the FEM with consequent reduction of the computational burden.

\subsection{FSHP applied to materials $A$ and $B$}

In this example we take into account both materials A (stiff grains) and B (soft grains) with elastic properties reported in Tab. 3. where also the contrast $c$ is reported, and the average dimension of grains and the hard-core have been fixed equal to $\bar{d}=40 \mu \mathrm{m}$ and $s=2 \mu \mathrm{m}$, respectively.

As an example, in Fig. 8 the variation of the average bulk modulus $\langle\mathbb{K}\rangle$ with respect to the number of simulations has been shown for different values of win- 


\begin{tabular}{|c|c|c|c|c|c|c|}
\hline \multicolumn{7}{|c|}{ Homogenized moduli } \\
\hline $\begin{array}{l}c \\
E_{i} / E_{m}\end{array}$ & Type & $\overline{\mathbb{C}}_{1111}^{D}$ & $\overline{\mathbb{C}}_{1122}^{D}$ & $\overline{\mathbb{C}}_{2211}^{D}$ & $\overline{\mathbb{C}}_{2222}^{D}$ & $\overline{\mathbb{C}}_{1212}^{D}$ \\
\hline \multirow{2}{*}{1} & VEM & 1.2000 & 0.4000 & 0.4000 & 1.2000 & 0.8000 \\
\hline & FEM & 1.2000 & 0.4000 & 0.4000 & 1.2000 & 0.8000 \\
\hline \multirow{2}{*}{2} & VEM & 2.0890 & 0.6869 & 0.6868 & 2.0929 & 1.3744 \\
\hline & FEM & 2.0909 & 0.6868 & 0.6869 & 2.0948 & 1.3767 \\
\hline \multirow{2}{*}{3} & VEM & 2.8116 & 0.9059 & 0.9060 & 2.8251 & 1.8140 \\
\hline & FEM & 2.8179 & 0.9059 & 0.9062 & 2.8313 & 1.8210 \\
\hline \multirow{2}{*}{4} & VEM & 3.4149 & 1.0791 & 1.0799 & 3.4411 & 2.1618 \\
\hline & FEM & 3.4265 & 1.0792 & 1.0804 & 3.4529 & 2.1739 \\
\hline \multirow{2}{*}{5} & VEM & 3.9287 & 1.2202 & 1.2223 & 3.9695 & 2.4438 \\
\hline & FEM & 3.9460 & 1.2204 & 1.2229 & 3.9873 & 2.4607 \\
\hline \multirow{2}{*}{6} & VEM & 4.3734 & 1.3377 & 1.3416 & 4.4296 & 2.6769 \\
\hline & FEM & 4.3965 & 1.3379 & 1.3423 & 4.4535 & 2.6981 \\
\hline \multirow{2}{*}{7} & VEM & 4.7634 & 1.4376 & 1.4437 & 4.8355 & 2.8728 \\
\hline & FEM & 4.7921 & 1.4377 & 1.4442 & 4.8653 & 2.8975 \\
\hline \multirow{2}{*}{8} & VEM & 5.1094 & 1.5237 & 1.5324 & 5.1973 & 3.0395 \\
\hline & FEM & 5.1435 & 1.5236 & 1.5327 & 5.2329 & 3.0671 \\
\hline \multirow{2}{*}{9} & VEM & 5.4193 & 1.5991 & 1.6106 & 5.5228 & 3.1830 \\
\hline & FEM & 5.4586 & 1.5986 & 1.6104 & 5.5638 & 3.2129 \\
\hline \multirow{2}{*}{10} & VEM & 5.6992 & 1.6657 & 1.6802 & 5.8180 & 3.3077 \\
\hline & FEM & 5.7434 & 1.6648 & 1.6794 & 5.8642 & 3.3395 \\
\hline
\end{tabular}

Table 1: Homogenized moduli obtained by applying Dirichlet boundary conditions 


\begin{tabular}{|c|c|c|c|c|c|c|}
\hline \multicolumn{7}{|c|}{ Homogenized moduli } \\
\hline $\begin{array}{l}c \\
E_{i} / E_{m}\end{array}$ & Type & $\overline{\mathbb{C}}_{1111}^{N}$ & $\overline{\mathbb{C}}_{1122}^{N}$ & $\overline{\mathbb{C}}_{2211}^{N}$ & $\overline{\mathbb{C}_{2222}^{N}}$ & $\overline{\mathbb{C}}_{1212}^{N}$ \\
\hline \multirow{2}{*}{1} & VEM & 1.2001 & 0.4003 & 0.4003 & 1.2001 & 0.8000 \\
\hline & FEM & 1.2001 & 0.4004 & 0.4004 & 1.2001 & 0.8000 \\
\hline \multirow{2}{*}{2} & VEM & 2.0971 & 0.6930 & 0.6930 & 2.1005 & 1.4637 \\
\hline & FEM & 2.0970 & 0.6927 & 0.6927 & 2.1006 & 1.4637 \\
\hline \multirow{2}{*}{3} & VEM & 2.8364 & 0.9215 & 0.9215 & 2.8488 & 2.1273 \\
\hline & FEM & 2.8361 & 0.9208 & 0.9208 & 2.8488 & 2.1273 \\
\hline \multirow{2}{*}{4} & VEM & 3.4588 & 1.1031 & 1.1031 & 3.4836 & 2.7910 \\
\hline & FEM & 3.4579 & 1.1021 & 1.1021 & 3.4832 & 2.7910 \\
\hline \multirow{2}{*}{5} & VEM & 3.9912 & 1.2496 & 1.2496 & 4.0303 & 3.4546 \\
\hline & FEM & 3.9894 & 1.2484 & 1.2484 & 4.0292 & 3.4546 \\
\hline \multirow{2}{*}{6} & VEM & 4.4525 & 1.3695 & 1.3695 & 4.5071 & 4.1183 \\
\hline & FEM & 4.4494 & 1.3684 & 1.3684 & 4.5047 & 4.1183 \\
\hline \multirow{2}{*}{7} & VEM & 4.8563 & 1.4688 & 1.4688 & 4.9269 & 4.7819 \\
\hline & FEM & 4.8517 & 1.4680 & 1.4680 & 4.9230 & 4.7819 \\
\hline \multirow{2}{*}{8} & VEM & 5.2131 & 1.5520 & 1.5520 & 5.2998 & 5.4456 \\
\hline & FEM & 5.2067 & 1.5516 & 1.5516 & 5.2941 & 5.4456 \\
\hline \multirow{2}{*}{9} & VEM & 5.5308 & 1.6223 & 1.6223 & 5.6335 & 6.1092 \\
\hline & FEM & 5.5226 & 1.6224 & 1.6224 & 5.6258 & 6.1092 \\
\hline \multirow{2}{*}{10} & VEM & 5.8155 & 1.6823 & 1.6823 & 5.9339 & 6.7729 \\
\hline & FEM & 5.8055 & 1.6830 & 1.6830 & 5.9241 & 6.7729 \\
\hline
\end{tabular}

Table 2: Homogenized moduli obtained by applying Neumann boundary conditions 


\begin{tabular}{llllll}
\hline Material & $c=E_{i} / E_{m}$ & $E_{m}[\mathrm{MPa}]$ & $E_{i}[\mathrm{MPa}]$ & $\nu_{m}$ & $\nu_{i}$ \\
\hline A10 & 10 & 1000 & 10000 & 0.25 & 0.23 \\
\hline A100 & 100 & 100 & 10000 & 0.25 & 0.23 \\
\hline B01 & 0.1 & 10000 & 1000 & 0.25 & 0.23 \\
\hline B001 & 0.01 & 10000 & 100 & 0.25 & 0.23 \\
\hline
\end{tabular}

Table 3: Mechanical properties adopted

number of simulations, required to archive the convergence, decreases. Accordingly to the convergence criterion adopted (see Section 2.2), the convergence is obtained for a given small value of the number of simulations, $N^{l i m}$.

In Figs. 9(a) and 9(b) the average values of the homogenized bulk modulus, $335\langle\mathbb{K}\rangle$, obtained for increasing window size, $L$, are reported.

The convergence trend for the different materials depends on the different dispersion of results, as shown in Fig. 10 and Fig. 11, where the Coefficient of Variation, $C V(\mathbb{K})$, is also plotted for several values of window size $L$ for Dirichlet (blue solid line) and Neumann (red solid line) BVPs.

\section{Final Remarks}

In this paper we present an efficient extension of the Fast Statistical Homogenization (FSHP), previously developed by the authors for the homogenization of particle composites in [36, 37, 69], to account for the peculiar geometry of the polycrystal and composites with grains and thin interfaces. The Virtual

345 Element Method is used as numerical tool to solve the partial differential equations governing the elastic problem. Starting from a random polygonal mesh, a novel automatic procedure to insert thin interfaces between grains is presented. Each grain is discretized using a single VEM, while the interfaces zones present a dense triangular mesh (exploiting Delaunay triangulation). Clear advantages in terms of computational burden are found with respect to the Finite Element Method (saving of about 350\% of degrees of freedom). This circumstances are particularly favourable in cases of materials with very high or very low contrasts, 


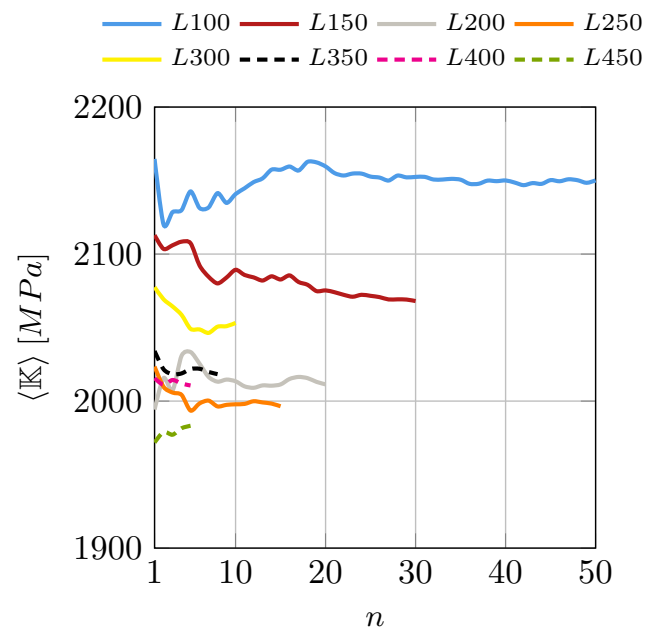

(a) Dirichlet boundary conditions

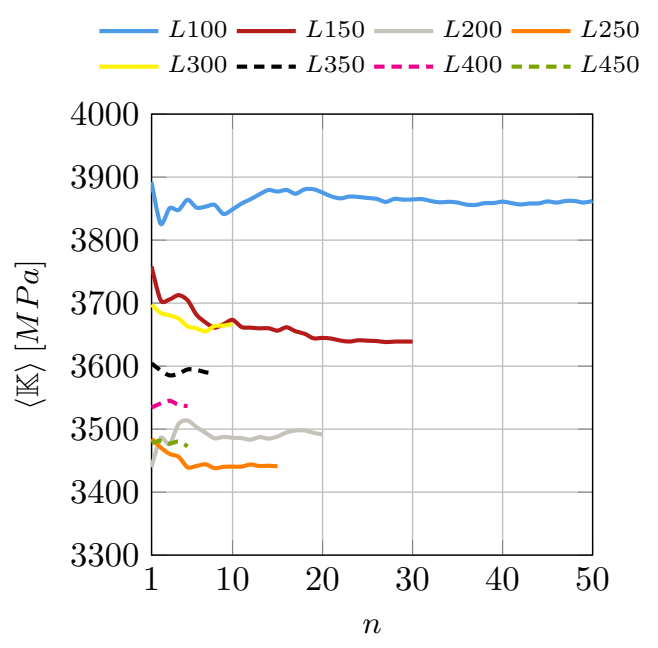

(b) Neumann boundary conditions

Figure 8: Material type B01: average bulk modulus $\langle\mathbb{K}\rangle$ versus the number of simulations $n$ for different window sizes $L$.

for which large window domains are required, as reported in [30, 19, 34, 9].

The results shown for a set of ideal materials, characterized by different mismatches between elastic moduli of grains and interfaces (material contrast), confirm the reliability and the efficiency of the statistical procedure in conjunc- 


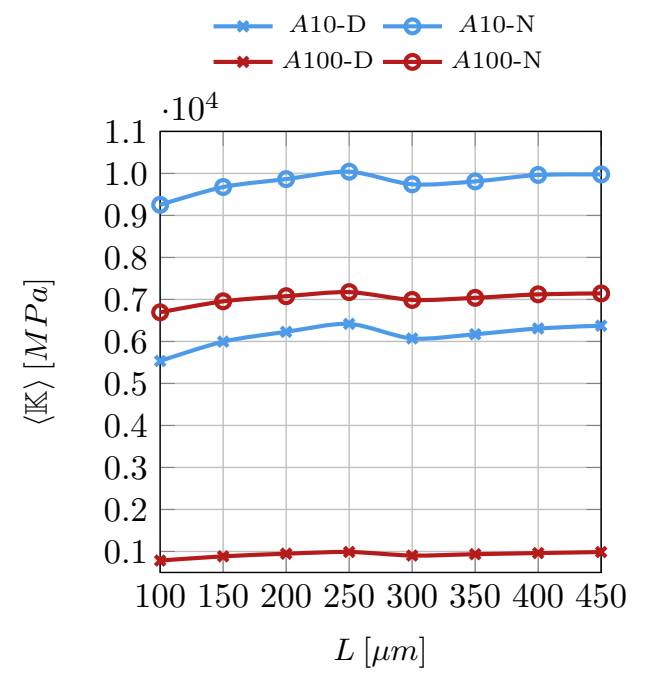

(a) Material type A

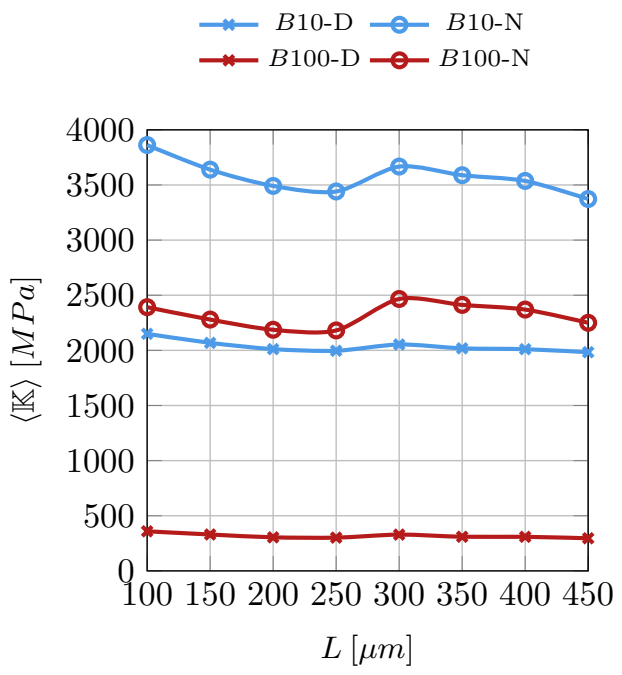

(b) Material type B

Figure 9: Average bulk modulus $\langle\mathbb{K}\rangle$ versus different window sizes $L$ : material type A (a) and material type B (b). 


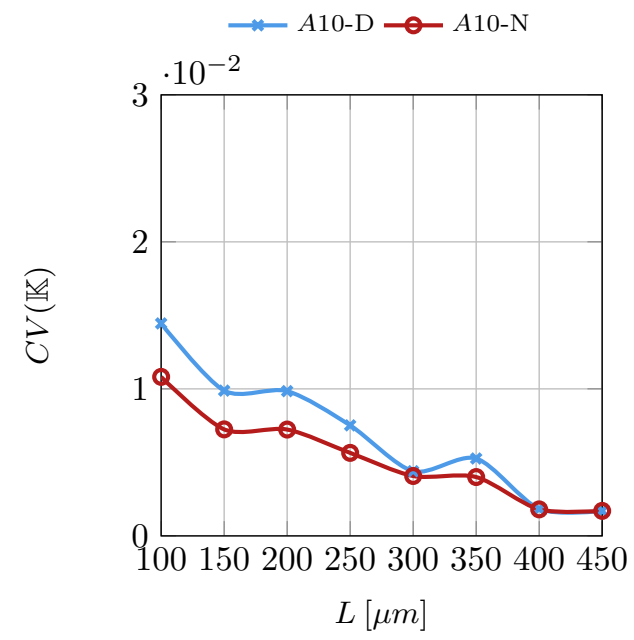

(a) Material type A10

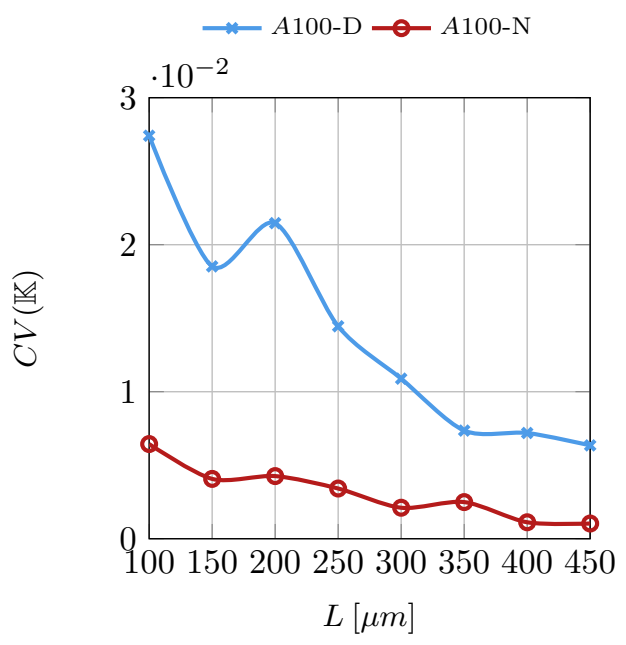

(b) Material type A100

Figure 10: Coefficient of variation $C V(\mathbb{K})$ for different window sizes $L$ : material type A.

tion with the innovative method of Virtual Elements Method.

Here, the analyses are limited to the Cauchy continuum and the extension to enriched non-classical continua, such as Couple-Stress and Cosserat, [34], in 


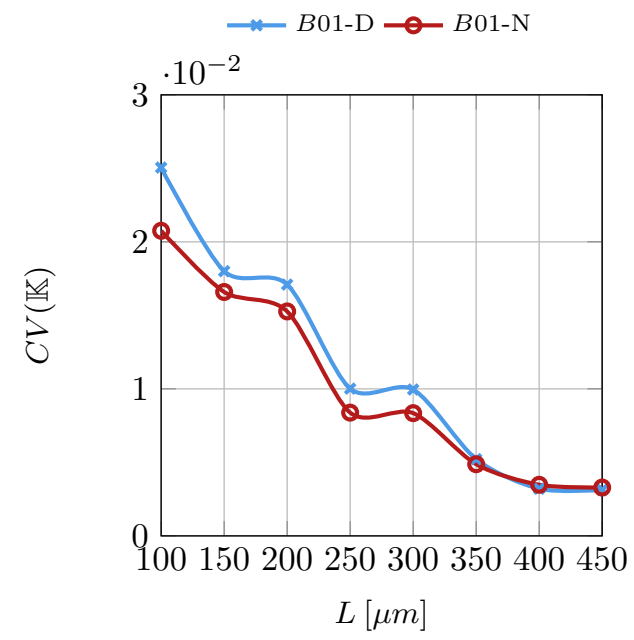

(a) Material type B01

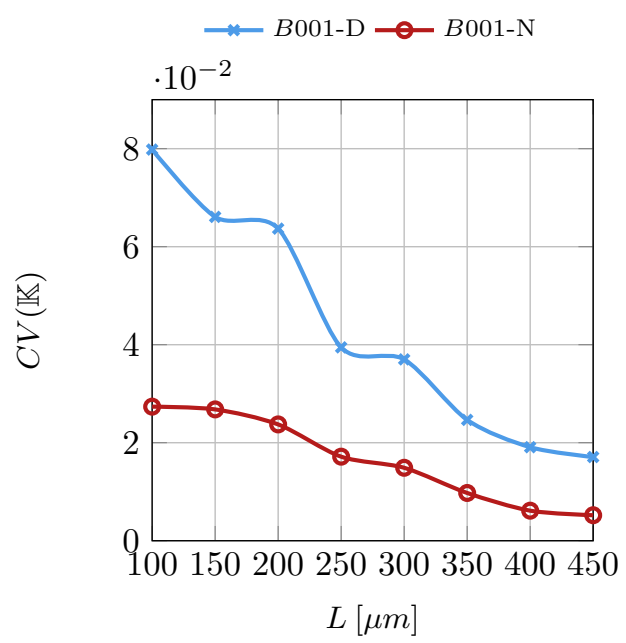

(b) Material type B001

Figure 11: Coefficient of variation $C V(\mathbb{K})$ for different window sizes $L$ : material type B.

360

order to better describe the behaviour of micro-structured material, is one of the future developments. Moreover, we will upgrade the procedure to take into account possible anisotropic behaviours, since this type of materials are gener- 
ally characterized by anisotropic properties, that varies grain-by-grain. Another interesting application and future development will be applying the procedure for the design of new materials with prescribed mechanical characteristics.

\section{Acknowledgements}

This work is supported by: Italian Ministry of University and Research PRIN 2017, project No. 2017HFPKZY (B88D19001130001); Sapienza Research Grants "Progetti Medi" 2017 (Grant No. B83C17001440005, ID: 10.13039/501100004271); Sapienza Research Grants "Progetti Grandi" 2018 (B81G19000060005).

\section{References}

\section{References}

[1] M. Boniecki, T. Sadowski, P. Gołębiewski, H. Węglarz, A. Piątkowska, M. Romaniec, K. Krzyżak, K. Łosiewicz, Mechanical properties of alumina/zirconia composites, Ceramics International 46 (1) (2020) 1033-1039. doi:10.1016/j.ceramint.2019.09.068.

[2] P. Golewski, A. Rusinek, T. Sadowski, Material characterization of $\mathrm{PMC} / \mathrm{TBC}$ composite under high strain rates and elevated temperatures, Materials 13 (1). doi:10.3390/ma13010167.

[3] T. Tosiriwatanapong, W. Singhatanadgit, Zirconia-Based Biomaterials for Hard Tissue Reconstruction, Bone and Tissue Regeneration Insights 9. doi:10.1177/1179061X18767886.

[4] M. Rosso, Ceramic and metal matrix composites: Routes and properties, 口 Journal of Materials Processing Technology 175 (1-3) (2006) 364-375. doi : $10.1016 / j \cdot j$ matprotec.2005.04.038.

[5] E. Postek, T. Sadowski, Impact model of WC/Co composite, Composite Structures 213 (2019) 231-242. doi:10.1016/j.compstruct.2019.01. 084 . 
[6] T. Sadowski, S. Hardy, E. Postek, Prediction of the mechanical response

[14] Y. Djebara, A. E. Moumen, T. Kanit, S. Madani, A. Imad, Modeling of the effect of particles size, particles distribution and particles number on mechanical properties of polymer-clay nano-composites: Numerical homogenization versus experimental results, Composites Part B: Engineering 86 415 of polycrystalline ceramics containing metallic intergranular layers under uniaxial tension, Computational Materials Science 34 (1) (2005) 46-63. doi:10.1016/j.commatsci.2004.10.005

[7] C. T. Herakovich, S. C. Baxter, Influence of pore geometry on the effective response of porous media, Journal of Materials Science 34 (7) (1999) 1595-

[8] A. P. Roberts, E. J. Garboczi, Elastic properties of model porous ceramics, Journal of the American Ceramic Society 83 (12) (2000) 3041-3048.

[9] E. Reccia, M. L. De Bellis, P. Trovalusci, R. Masiani, Sensitivity to material contrast in homogenization of random particle composites as micropolar continua, Composites Part B: Engineering 136 (2018) 39 - 45.

[10] M. Ostoja-Starzewski, Random Field Models of Heterogeneous Materials, International Journal of Solids and Structures 35 (19) (1998) 2429-2455.

[11] M. Ostoja-Starzewski, Microstructural Randomness and Scaling in Mechanics of Materials, CRC Press, Taylor \& Francis Group, 2007.

[12] T. Sadowski, L. Marsavina, Multiscale modelling of two-phase Ceramic Matrix Composites, Computational Materials Science 50 (4) (2011) 1336 1346.

[13] T. Sadowski, B. Pankowski, Numerical modelling of two-phase ceramic composite response under uniaxial loading, Composite Structures 143 (2016) 388 - 394.

(2016) $135-142$. 
[15] N. Fantuzzi, P. Trovalusci, R. Luciano, Multiscale analysis of anisotropic materials with hexagonal microstructure as micropolar continua, International Journal for Multiscale Computational Engineering 18 (2) (2020) 265284. doi:10.1615/Int JMultCompEng. 2020032920.

[20] T. Kanit, S. Forest, I. Galliet, V. Mounoury, D. Jeulin, Determination of the size of the representative volume element for random composites: statistical and numerical approach, International Journal of Solids and Structures 40 (13-14) (2003) $3647-3679$.

[21] X. Du, M. Ostoja-Starzewski, On the size of representative volume element for Darcy law in random media, Proceedings of the Royal Society of London A: Mathematical, Physical and Engineering Sciences 462 (2074) (2006) 2949-2963. 
[22] Z. F. Khisaeva, M. Ostoja-Starzewski, On the Size of RVE in Finite Elasticity of Random Composites, Journal of Elasticity 85 (2) (2006) 153.

445 [23] I. M. Gitman, H. Askes, L. J. Sluys, Representative volume: Existence and size determination, Engineering Fracture Mechanics 74 (16) (2007) 25182534.

[24] J. Zeman, M. Sejnoha, From random microstructures to representative volume elements, Modelling and Simulation in Materials Science and Engineering 15 (4) (2007) S325-S335.

[25] S. I. Ranganathan, M. Ostoja-Starzewski, Scaling Function, Anisotropy and the Size of RVE in Elastic Random Polycrystals, Journal of the Mechanics and Physics of Solids 56 (2008) 2773-2791.

[26] S. I. Ranganathan, M. Ostoja-Starzewski, Towards scaling laws in random polycrystals, International Journal of Engineering Science 47 (11) (2009) $1322-1330$.

[27] L. Bouaoune, Y. Brunet, A. E. Moumen, T. Kanit, H. Mazouz, Random versus periodic microstructures for elasticity of fibers reinforced composites, Composites Part B: Engineering 103 (2016) 68 - 73.

[28] D. Savvas, G. Stefanou, M. Papadrakakis, Determination of rve size for random composites with local volume fraction variation, Computer Methods in Applied Mechanics and Engineering 305 (2016) 340-358. doi: $10.1016 / \mathrm{j} . \mathrm{cma} .2016 .03 .002$

[29] D. Kubair, M. Pinz, K. Kollins, C. Przybyla, S. Ghosh, Role of exterior statistics-based boundary conditions for property-based statistically equivalent representative volume elements of polydispersed elastic composites., Journal of Composite Materials.

[30] P. Trovalusci, M. L. De Bellis, M. Ostoja-Starzewski, A. Murrali, Particulate random composites homogenized as micropolar materials, Meccanica 49 (11) (2014) 2719-2727. 
[31] M. Ostoja-Starzewski, Material spatial randomness: From statistical to representative volume element, Probabilistic Engineering Mechanics 21 (2006) 112-132.

[32] R. Hill, Elastic properties of reinforced solids: Some theoretical principles, Journal of the Mechanics and Physics of Solids 11 (5) (1963) 357 - 372.

[33] P. Trovalusci, M. De Bellis, M. Ostoja-Starzewski, A statistically-based homogenization approach for particle random composites as micropolar continua, Advanced Structured Materials 42 (2016) 425-441, cited By 8. doi:10.1007/978-3-319-31721-2_20

${ }_{480}$ [34] P. Trovalusci, M. L. De Bellis, R. Masiani, A multiscale description of particle composites: From lattice microstructures to micropolar continua, Composites Part B: Engineering 128 (2017) $164-173$.

[35] M. Salmi, F. Auslender, M. Bornert, M. Fogli, Apparent and effective mechanical properties of linear matrix-inclusion random composites: Improved bounds for the effective behavior, International Journal of Solids and Structures 49 (10) (2012) 1195 - 1211.

[36] M. Pingaro, E. Reccia, P. Trovalusci, R. Masiani, Fast statistical homogenization procedure (FSHP) for particle random composites using virtual element method, Computational Mechanics 64 (1) (2019) 197-210. doi : 10.1007/s00466-018-1665-7.

[37] M. Pingaro, E. Reccia, P. Trovalusci, Homogenization of Random Porous Materials With Low-Order Virtual Elements, ASCE-ASME Journal of Risk and Uncertainty in Engineering Systems, Part B: Mechanical Engineering 5 (3). doi:10.1115/1.4043475

495 [38] L. Beirão Da Veiga, F. Brezzi, A. Cangiani, G. Manzini, L. Marini, A. Russo, Basic principles of virtual element methods, Mathematical 【 Models and Methods in Applied Sciences 23 (1) (2013) 199-214. doi: $10.1142 / \mathrm{S} 0218202512500492$ 
[39] L. Beirão Da Veiga, F. Brezzi, L. Marini, Virtual elements for linear elastic-

[40] M. Mengolini, M. Benedetto, A. Aragón, An engineering perspective to the virtual element method and its interplay with the standard finite element method, Computer Methods in Applied Mechanics and Engineering 350

[41] E. Artioli, L. Beirão da Veiga, F. Dassi, Curvilinear Virtual Elements for 2D solid mechanics applications, Computer Methods in Applied Mechanics and Engineering 359. doi:10.1016/j.cma.2019.112667.

[42] E. Benvenuti, A. Chiozzi, G. Manzini, N. Sukumar, Extended virtual element method for the Laplace problem with singularities and discontinuities, Computer Methods in Applied Mechanics and Engineering 356 (2019) 571597. doi:10.1016/j.cma.2019.07.028.

[43] A. L. Gain, C. Talischi, G. H. Paulino, On the virtual element method for three-dimensional linear elasticity problems on arbitrary polyhedral meshes, Computer Methods in Applied Mechanics and Engineering 282 (2014) 132-160.

[44] D. B. Reddy, D. van Huyssteen, A virtual element method for transversely

[45] E. Artioli, S. de Miranda, C. Lovadina, L. Patruno, A stress/displacement Virtual Element method for plane elasticity problems, Computer Methods घ in Applied Mechanics and Engineering 325 (2017) 155-174. doi:10.1016/ j.cma.2017.06.036.

[46] P. Wriggers, B. Hudobivnik, A low order virtual element formulation for 525 isotropic elasticity, Computational Mechanics 64 (2019) 971-988. doi: $10.1007 / \mathrm{s} 00466-019-01690-7$. finite elasto-plastic deformations, Computer Methods in Applied Mechanics and Engineering 327 (2017) 459-477. 
[47] M. De Bellis, P. Wriggers, B. Hudobivnik, Serendipity virtual element formulation for nonlinear elasticity, Computers and Structures 223. doi: $10.1016 / j$. compstruc.2019.07.003

[48] R. Taylor, E. Artioli, VEM for inelastic solids, Computational Methods in Applied Sciences 46 (2018) 381-394. doi:10.1007/978-3-319-60885-3_ 18.

[49] K. Park, H. Chi, G. Paulino, On nonconvex meshes for elastodynamics using virtual element methods with explicit time integration, Computer Methods in Applied Mechanics and Engineering 356 (2019) 669-684. doi: $10.1016 / \mathrm{j} . \mathrm{cma} .2019 .06 .031$

[50] P. Antonietti, M. Bruggi, S. Scacchi, M. Verani, On the virtual element method for topology optimization on polygonal meshes: A numerical study, Computers and Mathematics with Applications 74 (5) (2017) 1091-1109. doi:10.1016/j.camwa.2017.05.025

[51] H. Chi, A. Pereira, I. Menezes, G. Paulino, Virtual element method (VEM)based topology optimization: an integrated framework, Structural and Multidisciplinary Optimizationdoi:10.1007/s00158-019-02268-w

[52] P. Wriggers, W. Rust, B. Reddy, A virtual element method for con545 tact, Computational Mechanics 58 (6) (2016) 1039-1050. doi:10.1007/ s00466-016-1331-x.

[53] F. Brezzi, L. Marini, Virtual Element Methods for plate bending problems, Computer Methods in Applied Mechanics and Engineering 253 (2013) 455462. doi:10.1016/j.cma.2012.09.012.

550 [54] M. Benedetto, A. Caggiano, G. Etse, Virtual elements and zero thickness interface-based approach for fracture analysis of heterogeneous materials, Computer Methods in Applied Mechanics and Engineering 338 (2018) 4167. doi:10.1016/j.cma.2018.04.001. 
[55] M. L. De Bellis, P. Wriggers, B. Hudobivnik, G. Zavarise, Virtual element formulation for isotropic damage, Finite Elements in Analysis and Design 144 (2018) $38-48$.

[56] E. Artioli, S. Marfia, E. Sacco, High-order virtual element method for the homogenization of long fiber nonlinear composites, Computer Methods in Applied Mechanics and Engineering 341 (2018) 571-585. doi:10.1016/j. cma.2018.07.012.

[57] E. Artioli, L. Beirão da Veiga, M. Verani, An adaptive curved virtual element method for the statistical homogenization of random fibre-reinforced composites, Finite Elements in Analysis and Design 177 (2020) 103418. doi:10.1016/j.finel.2020.103418

[58] M. Marino, B. Hudobivnik, P. Wriggers, Computational homogenization of polycrystalline materials with the Virtual Element Method, Computer

口 Methods in Applied Mechanics and Engineering 355 (2019) 349-372. doi: $10.1016 /$ j.cma.2019.06.004

[59] P. Trovalusci, M. Ostoja-Starzewski, M. De Bellis, A. Murrali, Scale570 dependent homogenization of random composites as micropolar continua European Journal of Mechanics, A/Solids 49 (2015) 396-407. doi:10.1016/j.euromechsol.2014.08.010

11 URL https://www.scopus.com/inward/record.uri?eid=2-s2.

10-84907906950\&doi=10.1016\%2f j.euromechsol.2014.08.010\&

575 partnerID=40\&md5=7477863fa210a4512e574c0551a85225

[60] P. Trovalusci, M. De Bellis, M. Ostoja-Starzewski, A. Murrali, Particulate random composites homogenized as micropolar materials, Meccanica 49 (11) (2014) 2719-2727. doi:10.1007/s11012-014-0031-x.

a URL https://www.scopus.com/inward/record.uri?eid=2-s2.

$580 \quad 0-84911992445 \& d o i=10.1007 \% 2 f \mathrm{~s} 11012-014-0031-x \& p a r t n e r I D=$ $40 \& m d 5=20$ be 8 aea $1 \mathrm{f} 07837 \mathrm{c} 9 \mathrm{e} 740 \mathrm{e} 4 \mathrm{ae} 9 \mathrm{df} 57 \mathrm{~b} 3$ 
[61] B. M. Said, M. Salah, T. Kanit, F. Kamel, On the homogenization of 2d porous material with determination of rve, International Journal of Mechanical and Mechatronics Engineering 16 (1) (2016) 81-86.

[62] C. Talischi, G. Paulino, A. Pereira, I. Menezes, PolyMesher: A generalpurpose mesh generator for polygonal elements written in Matlab, Structural and Multidisciplinary Optimization 45 (3) (2012) 309-328. doi: $10.1007 / \mathrm{s} 00158-011-0706-\mathrm{z}$

[63] J. R. Shewchuk, Delaunay refinement algorithms for triangular mesh generation, Computational Geometry 22 (1) (2002) $21-74$.

[64] L. Da Veiga, F. Brezzi, L. Marini, A. Russo, F. Brezzi, G. Manzini, The Hitchhiker's guide to the virtual element method, Mathematical Models and Methods in Applied Sciences 24 (8) (2014) 1541-1573. doi:10.1142/ S021820251440003X.

[65] E. Artioli, L. Beirão da Veiga, C. Lovadina, E. Sacco, Arbitrary order $2 \mathrm{~d}$ virtual elements for polygonal meshes: part i, elastic proba lem, Computational Mechanics 60 (3) (2017) 355-377. doi:10.1007/ s00466-017-1404-5.

[66] V. Nguyen-Thanh, X. Zhuang, H. Nguyen-Xuan, T. Rabczuk, P. Wriggers, A Virtual Element Method for 2D linear elastic fracture analysis, Computer

n Methods in Applied Mechanics and Engineering 340 (2018) 366-395. doi: $10.1016 / \mathrm{j} . \mathrm{cma} \cdot 2018.05 .021$

[67] M. Paggi, E. Lehmann, C. Weber, A. Carpinteri, P. Wriggers, M. Schaper, A numerical investigation of the interplay between cohesive cracking and plasticity in polycrystalline materials, Computational Materials Science 77 (2013) 81-92. doi:10.1016/j.commatsci.2013.04.002.

[68] A. Sreekumar, S. Triantafyllou, F.-X. Bécot, F. Chevillotte, A multiscale virtual element method for the analysis of heterogeneous media, Interna- 
tional Journal for Numerical Methods in Engineering 121 (8) (2020) 1791-

[69] M. Pingaro, E. Reccia, P. Trovalusci, M. De Bellis, Statistical homogeniza-

a tion of random porous media, 2019, pp. 590-599. doi:10.7712/120219. 6362.18857 . 